\title{
Emergence Percentage and Speed of Rootstocks for Citriculture in South of Brazil
}

\author{
Léo O. D. Marques ${ }^{1}$, Paulo Mello-Farias ${ }^{1}$, Roberto P. de Oliveira $^{2}$, Maximiliano Dini ${ }^{1}$, Walter S. Soares Filho ${ }^{3}$ \\ \& Marcelo B. Malgarim ${ }^{1}$ \\ ${ }^{1}$ Departament of Horticulture and Crop Science, Faculty of Agronomy Eliseu Maciel, Federal University of \\ Pelotas, Pelotas, Rio Grande do Sul, Brazil \\ ${ }^{2}$ Embrapa Temperate Climate, Pelotas, Rio Grande do Sul, Brazil \\ ${ }^{3}$ Embrapa Cassava and Fruit Science, Cruz das Almas, Bahia, Brazil \\ Correspondence: Léo O. D. Marques, Federal University of Pelotas, Faculty of Agronomy Eliseu Maciel, \\ Departament of Horticulture and Crop Science, Campus Capão do Leão, s/n, 96160-000, Rio Grande do Sul, \\ Brazil. Tel: 55-51-996-381-893. E-mail: leodmq@hotmail.com
}

$\begin{array}{ll}\text { Received: January 21, } 2019 & \text { Accepted: February 27, } 2019 \quad \text { Online Published: April 15, } 2019 \\ \text { doi:10.5539/jas.v11n5p49 } & \text { URL: https://doi.org/10.5539/jas.v11n5p49 }\end{array}$

This study was financed in part by the Coordenação de Aperfeiçoamento de Pessoal de Nível Superior-Brazil (CAPES)-Finance Code 001.

\begin{abstract}
The fact that Brazilian citriculture has focused on few rootstocks poses several phytosanitary risks to the culture and has made genetic improvement programs develop hybrid genotypes to be used as novel alternatives. This study aimed at evaluating the behavior of 42 different rootstocks regarding their emergence percentage and speed in weather conditions experienced in the extreme south of Brazil. Seeds of hybrids developed by the Citrus Genetic Improvement Program (PMG Citros) at the Embrapa Mandioca e Fruticultura (PMG Citros) and cultivars from other citrus growing regions were sown in conical tubes filled with commercial substrate in May 2017. A thoroughly randomized design with 4 replicates, each composed of 45 sampling units, was employed. Emergence percentage and emergence speed of every genotype were evaluated and the ones with the highest emergence percentage of seedlings and the ones with the most precocious emergence were identified. All genotypes completed seedling emergence 98 days after sowing. Seeds of Trifoliata, TSKC $\times$ CTSW-041 and TSKC $\times$ CTSW-025 had the highest values of seedling emergence whereas the highest emergence speed indexes were exhibited by genotypes Trifoliata and lemon tree 'Cravo'.
\end{abstract}

Keywords: adaptation, Citrus spp., diversification, improvement, seedlings

\section{Introduction}

Brazilian citriculture is very relevant in the global scenario, mainly the production of sweet oranges [Citrus sinensis (L.) Osbeck]. Brazil yields 16240000 ton annually and is the world's largest producer of this fruit. China ranks second, with 7823550 ton per year while India ranks third, with 7313610 ton per year (FAO, 2014). The citrus chain in Rio Grande do Sul state comprises about 20 thousand producers; most are families that grow citrus in almost 40 thousand hectares and play an important socio-economic role in the state (Oliveira et al., 2016).

Citrus production in Rio Grande do Sul focuses on Trifoliata [Poncirus trifoliata (L.) Raf.], since this rootstock is tolerant to cold and induces the production of high quality fruits (Petry et al., 2015). The agroclimatic zoning of citrus recommends the use of rootstocks which are tolerant to cold in four out of five citrus growing regions in the state. It explains the predominance of Trifoliata as a rootstock (Wrege et al., 2004).

History shows that Brazilian citriculture went through some crisis due to the fact that it has focused on few rootstocks. For instance, millions of orange trees 'Caipira' $(C$. sinensis) died at the beginning of the 20th century because this rootstock is susceptible to both gummosis (Phytophthora spp.) and water deficit. Besides, orange trees 'Azeda' (C. aurantium L.) died at the end of the 1930' and the beginning of the 1940's due to their 
susceptibility to the citrus tristeza virus (CTV). At the beginning of the 2000's, citrus sudden death (CSD) affected several orchards grafted on lemon trees 'Cravo' in the north and northwest of São Paulo state and in the south of a region named Triângulo Mineiro, in Minas Gerais state. Hence, the need for rootstock diversification (Oliveira et al., 2008).

Studies have tried to find solutions to problems related to biotic and abiotic stresses and the most desirable agronomic characteristics by means of genetic improvement. Thus, the Citrus Genetic Improvement Program (PMG Citros) at the Embrapa Mandioca e Fruticultura has developed varietal alternatives, such as rootstocks, in Brazilian citriculture (Rodrigues et al., 2015). Genetic materials developed by these studies need to be evaluated in different agroecosystems so that the best rootstocks for each region can be determined.

Commercial production of citrus rootstocks has been basically carried out by means of seeds. This method has been widely used because most species of genera Citrus L., Poncirus L. and Fortunella Swing are polyembryonic. It enables propagation of genetic material, which is identical to the matrix plant, from seed nucellar tissue (Oliveira et al., 2016).

It should also be taken into account that citrus seedlings are the main input to establish an orchard and that a phytosanitary approach is important because of the wide variety of diseases and plagues that affect citriculture. Therefore, it is important to yield seedlings in certified nurseries, i.e., in a protected environment named screened nursery (Oliveira et al., 2008; Sarmiento et al., 2016; Parolin et al., 2017).

Production of citrus seedlings in screened nurseries occurs between eight and 24 months. It depends mainly on the vigor of the rootstock and on local weather conditions. In order to optimize nurserymen's profit, rootstocks that exhibit vigor and speed, from seedling emergence to grafting, are preferable (Hayashi et al., 2012; Girardi et al., 2017; Oliveira et al., 2017).

This study aimed at evaluating the behavior of 42 rootstocks regarding seedling emergence and emergence speed index in weather conditions experienced in the extreme south of Brazil, so as to find new alternative rootstocks for citriculture in the region.

\section{Method}

\subsection{Location and Climate of the Region}

The experiment was carried out in a glass greenhouse at the Embrapa Clima Temperado in Pelotas, RS, Brazil (31 $40^{\prime} 47^{\prime \prime} \mathrm{S}$ and $52^{\circ} 26^{\prime} 24^{\prime \prime} \mathrm{W}$; at $57 \mathrm{~m}$ of altitude). In the Köppen climate classification, the climate in the region is humid-mesothermal subtropical $(\mathrm{Cfb})$, with no dry season and with moderate winters. Mean annual temperature is $17.8^{\circ} \mathrm{C}$ while mean annual rainfall is $1,367 \mathrm{~mm}$ and mean air relative humidity is $80.7 \%$.

\subsection{Sowing: Procedures, Substrate and Temperature}

Sowing was carried out in $50 \mathrm{~cm}^{3}$ plastic conical tubes, which had four longitudinal incisions and a drain and were suspended from a metallic workbench. They were filled with the commercial substrate Turfa Fértil at $\mathrm{pH}$ 5.8 ; electrical conductivity was $0.7 \mathrm{mS} / \mathrm{cm}$, maximum humidity was $55 \%$, dry matter density was $260 \mathrm{~kg} / \mathrm{m}^{3}$ and water retention capacity was $60 \%$. Before the substrate was placed in the tubes, $10.5 \mathrm{~g}$ slow-release fertilizer (Osmocote 15-10-10) per kg substrate was added to it.

Sowing was carried out in May 2017 at a depth of $1.5 \mathrm{~cm}$, a seed per tube (Figure 1). Temperature inside the greenhouse was kept at $25^{\circ} \mathrm{C}$ by electric heaters, from sowing to the end of the emergence period. 


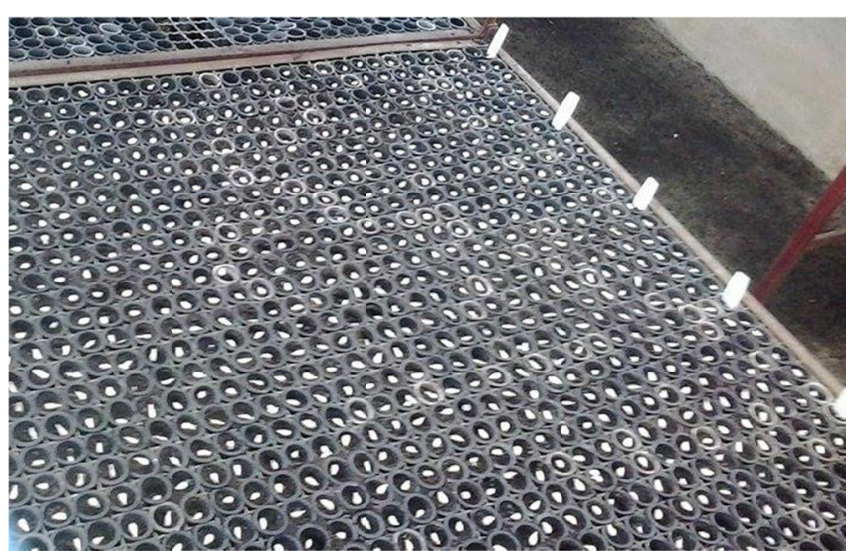

Figure 1. Seeds from different genotypes of citrus rootstocks before being covered with substrate at a depth of $1.5 \mathrm{~cm}$

\subsection{Seedlings Irrigation and Management}

Daily watering was carried out by a system of a plastic hose with a shower at one end (Figure 2). Seedlings were thinned out every four days so as to leave only one plant per tube and eliminate hybrid individuals. The ones of nucellar origin were maintained since they have their parents' genetic constitution.

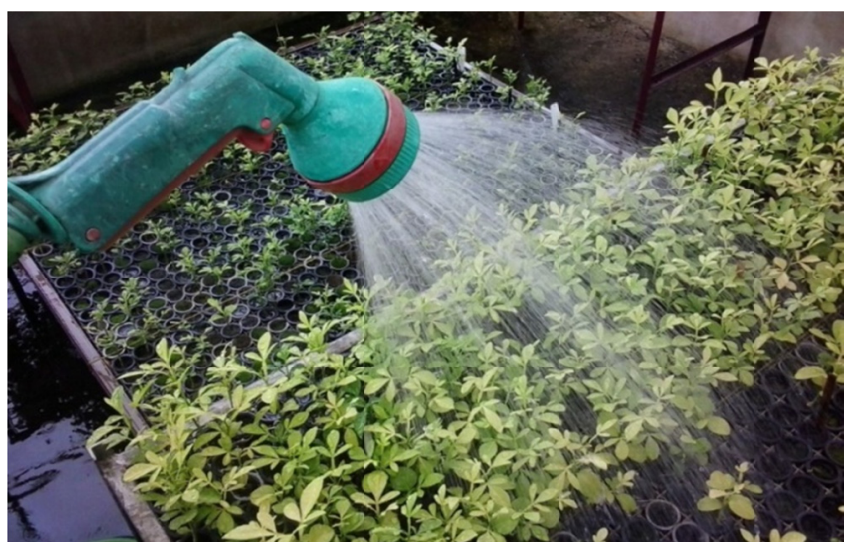

Figure 2. Different genotypes of citrus rootstocks, being irrigated by hose system with shower at the end

\subsection{Variables Under Study}

Emergence percentage: this variable was determined by counting emergent seedlings every three days.

Emergence speed index (ESI): it is the number of seedlings which emerges per time unit. It is determined by the formula $\mathrm{ESI}=\mathrm{G} 1 / \mathrm{N} 1+\mathrm{G} 2 / \mathrm{N} 2+\mathrm{Gn} / \mathrm{Nn}$, where, G1, G2 and Gn are the numbers of emerged seedlings which were found in the first count, second count, up to the last count, 98 days after sowing (DAS). N1, N2 and Nn are the numbers of days of the first count, second count, up to the last one (Maguirre, 1962; Rodrigues et al., 2015). The total emergence period was divided in 0-56 days after sowing and 57-98 days after sowing, since the use of the ESI found in the whole period did not enable to identify which rootstocks had the most precocious emergence.

Performance of hybrids per parents: division in groups of hybrids was based on parents tangerine tree 'Sunki' [ $C$. sunki (Hayata) hort. ex Tanaka], lemon tree 'Cravo' (C. limonia Osbeck) and Trifoliata (P. trifoliata). Since many hybrids originated from crossings among these parents, a hybrid could be in two or more groups, i.e., every hybrid that had certain parent in its constitution, either directly or indirectly, was included in its parent's group.

\subsection{Plant Material and Experimental Design}

Forty-two genotypes were studied (Table 1), by a thoroughly randomized design in four blocks, each composed of 45 sampling units. 
Table 1. List of rootstocks under study

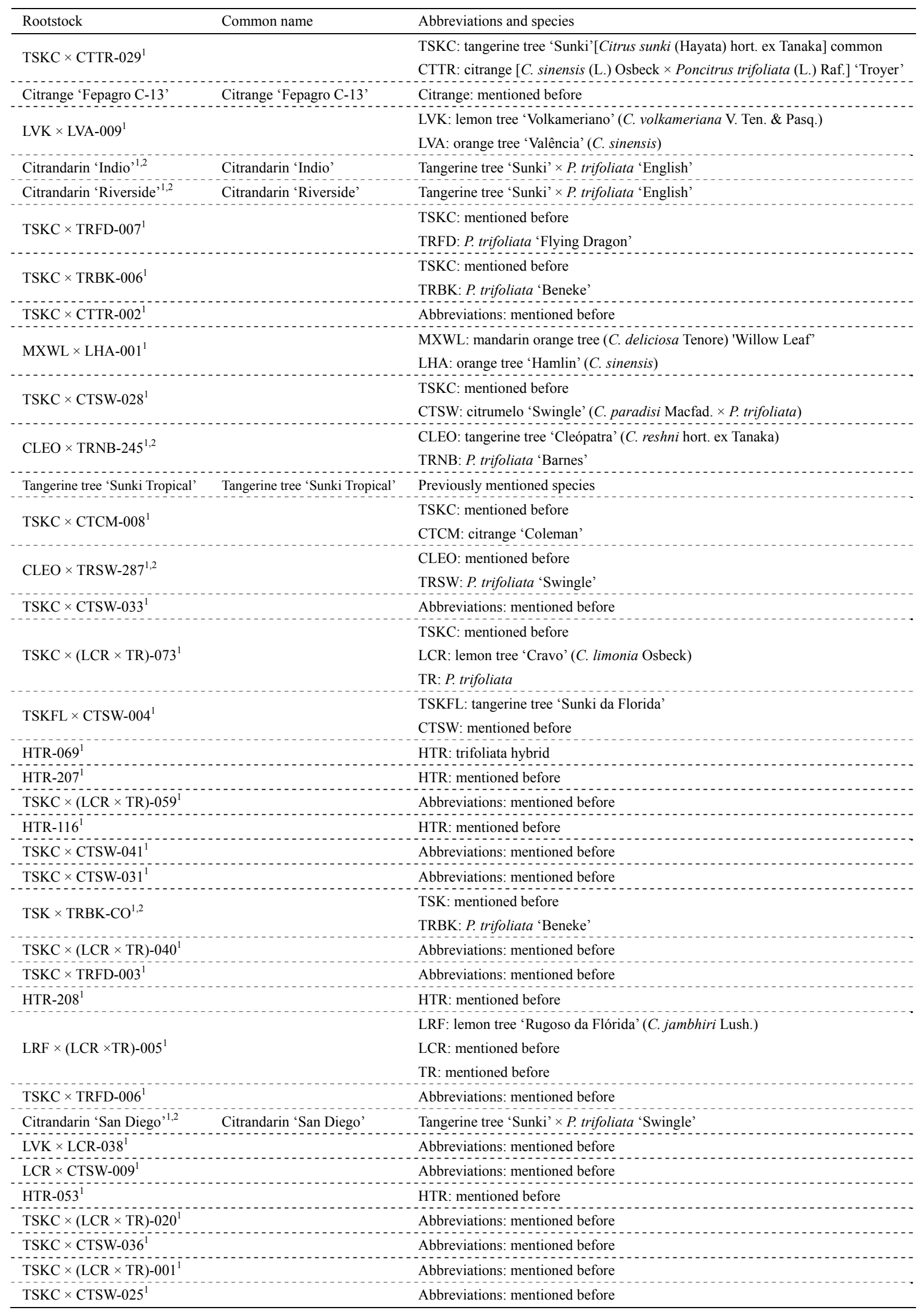




\begin{tabular}{|c|c|c|}
\hline $\mathrm{LCR} \times \mathrm{TR}-001^{1}$ & & Abbreviations: mentioned before \\
\hline TSKC $\times$ TRFD $-007^{1}$ & & Abbreviations: mentioned before \\
\hline HTR-051 & & HTR: mentioned before \\
\hline Trifoliata & Trifoliata & P. trifoliata \\
\hline Citrumelo 'Swingle' & Citrumelo 'Swingle' & C. paradisi $\times P$. trifoliata \\
\hline Lemon tree 'Cravo' & Lemon tree 'Cravo' & Previously mentioned species \\
\hline
\end{tabular}

Note. ${ }^{1}$ Hybrid selected by the Citrus Genetic Improvement Program (PMG Citros) at the Embrapa Mandioca $\mathrm{e}$ Fruticultura, Cruz das Almas, BA, Brazil.

${ }^{2}$ Hybrid introduced by the United States Department of Agriculture (USDA).

\subsection{Statistical Analysis}

Statistical analyses were carried out by the Infostat program. The analysis of variance (ANOVA) was conducted by the F-test. When they were meaningful, data were submitted to the Scott-Knott's clustering test (Scott \& Knott, 1974) at $5 \%$ level of significance. Percentages were transformed by the expression: $\arcsin \sqrt{\mathrm{x} / 100}$, where, $\mathrm{x}$ is the value of replicates of every response variable. This transformation was carried out to meet the assumption of variance homogeneity claimed by ANOVA. However, results are shown in the original scale of these variables.

\section{Results and Discussion}

All genotypes needed 98 days (after sowing) to complete seedling emergence. Trifoliata and both hybrids TSKC $\times$ CTSW-041 and TSKC $\times$ CTSW-025 did not differ statistically from each other; they had the highest seedling emergence (Table 2). Passos et al. (2006), Oliveira and Scivittaro (2007) and Sarmiento et al. (2016) found lower percentages of seedling emergence for the rootstock Trifoliata, i.e., $81 \%, 84.4 \%$ and $80 \%$, respectively.

According to Oliveira and Scivittaro (2007), the ideal temperature for Trifoliata seedling emergence is about $25{ }^{\circ} \mathrm{C}$. This factor may have triggered the excellent performance of the rootstock. The period of seedling emergence took place at the beginning of winter; thus, the need to raise the temperature in the greenhouse to $25^{\circ} \mathrm{C}$. If sowing had been carried out at higher temperatures, i.e., if temperature in the greenhouse were above $25^{\circ} \mathrm{C}$, rootstocks from hot regions, such as lemon tree 'Cravo' and tangerine tree 'Sunki' [C. sunki (Hayata) hort. ex Tanaka], as well as their hybrids, might have exhibited higher seedling emergence.

Hybrid TSKC $\times$ CTSW-041, whose seedling emergence was $92.2 \%$ (Table 2), had high values in this variable, in studies carried out by Rodrigues et al. (2015) and Parolin et al. (2017). They found seedling emergence of $100 \%$ and $98 \%$, respectively, and considered it a genotype whose seeds had high viability. Another rootstock that had good performance in seedling emergence was TSKC $\times$ CTSW-025 (Table 2). Parolin et al. (2017) found values of $77 \%$ while the study reported by this paper found $87.8 \%$. Variations in emergence percentages may have happened either because of the quality of the seeds or because different substrates were used.

Both hybrids LCR $\times$ TR-001 and TSKC $\times$ CTCM-008 exhibited seedling emergence values below $25 \%$ (Table 2), the lowest values found by this experiment. Among rootstocks that had the lowest emergence values, LCR $\times$ TR-001 stands out because it had the lowest performance in studies carried out by Rodrigues et al. (2015) and Parolin et al. (2017). They found $47 \%$ and $46 \%$, respectively, confirmed that this genotype does not exhibit good seedling emergence in different weather conditions and warned that the number of seeds per tube needs to be increased in the sowing season.

Lemon tree 'Cravo', which is one of the most common rootstocks in Brazilian citriculture, was one of the five genotypes that had the highest seedling emergence, i.e., 83.9\% (Table 2). Passos et al. (2006) found that seeds of lemon tree 'Cravo' had 74\% seedling emergence while Rodrigues et al. (2015) found 90\% in studies conducted in the same places and times of the year. It shows that variation in seedling emergence may be related either to the use of different substrates or to the seed quality of the genotype used in the experiments.

Final seedling emergence of citrumelo 'Swingle' $(C$. paradisi Macfad. $\times$ P. trifoliata) was $66.1 \%$ (Table 2). It is relatively low and agrees with results of other studies carried out in the weather conditions found in Rio Grande do Sul. The citrumelo 'Swingle' usually has low values of seedling emergence. It was observed by several studies, such as the ones conducted by Teixeira et al. (2009), Rodrigues et al. (2015) and Sarmiento et al. (2016), who found $51 \%, 79 \%$ and $65 \%$, it means that more seeds need to be sown per tube, with the purpose of avoiding losses in the next stages of seedling production, due to the low emergence percentage of the rootstock seeds. 
Table 2. Emergence percentage of seedlings at 19, 34, 50, 65, 80 and 98 days after sowing (DAS)

\begin{tabular}{|c|c|c|c|c|c|c|}
\hline \multirow{2}{*}{ Rootstock } & \multicolumn{6}{|c|}{ Emergence percentage of seedlings (\%) } \\
\hline & 19 DAS & $34 \mathrm{DAS}$ & $50 \mathrm{DAS}$ & 65 DAS & 80 DAS & $98 \mathrm{DAS}$ \\
\hline TSKC $\times$ CTSW -025 & $0 \mathrm{c}$ & $2.8 \mathrm{~d}$ & $31.1 \mathrm{~d}$ & $86.1 \mathrm{a}$ & $87.2 \mathrm{a}$ & $87.8 \mathrm{a}$ \\
\hline TSKC $\times$ CTSW- 028 & $0 \mathrm{c}$ & $0.5 \mathrm{e}$ & $7.0 \mathrm{f}$ & $35.3 \mathrm{e}$ & $39.1 \mathrm{~d}$ & $44.0 \mathrm{~d}$ \\
\hline TSKC $\times$ CTSW -031 & $1.1 \mathrm{~b}$ & $7.2 \mathrm{c}$ & $27.8 \mathrm{~d}$ & $48.9 \mathrm{c}$ & $50.0 \mathrm{~d}$ & $51.1 \mathrm{~d}$ \\
\hline TSKC $\times$ CTSW-033 & $0.6 \mathrm{~b}$ & $1.7 \mathrm{~d}$ & $11.1 \mathrm{e}$ & $40.6 \mathrm{~d}$ & $56.1 \mathrm{~d}$ & $63.3 \mathrm{c}$ \\
\hline TSKC $\times$ CTSW-036 & $0 \mathrm{c}$ & $0 \mathrm{e}$ & $1.7 \mathrm{~g}$ & $18.3 \mathrm{f}$ & $26.1 \mathrm{e}$ & $32.8 \mathrm{e}$ \\
\hline TSKC $\times$ CTSW-041 & $0.6 \mathrm{~b}$ & $7.8 \mathrm{c}$ & $30.6 \mathrm{~d}$ & $85.6 \mathrm{a}$ & $91.1 \mathrm{a}$ & $92.2 \mathrm{a}$ \\
\hline TSKC $\times$ TRFD-003 & $0 \mathrm{c}$ & $6.1 \mathrm{c}$ & $38.9 \mathrm{c}$ & $79.4 \mathrm{a}$ & $83.3 \mathrm{~b}$ & $83.3 \mathrm{~b}$ \\
\hline TSKC $\times$ TRFD-006 & $0 \mathrm{c}$ & $6.7 \mathrm{c}$ & $20.0 \mathrm{e}$ & $55.0 \mathrm{c}$ & $65.0 \mathrm{c}$ & $68.9 \mathrm{c}$ \\
\hline TSKC $\times$ TRFD-007 & $0 \mathrm{c}$ & $4.3 \mathrm{~d}$ & $18.1 \mathrm{e}$ & $50.9 \mathrm{c}$ & $69.0 \mathrm{c}$ & $71.6 \mathrm{c}$ \\
\hline $\mathrm{TSKC} \times \mathrm{CTCM}-008$ & $0 \mathrm{c}$ & $0 \mathrm{e}$ & $1.7 \mathrm{~g}$ & $8.9 \mathrm{~g}$ & $16.7 \mathrm{e}$ & $20.0 \mathrm{f}$ \\
\hline TSKC $\times$ CTTR-002 & $1.6 \mathrm{~b}$ & $7.1 \mathrm{c}$ & $25.5 \mathrm{~d}$ & $67.9 \mathrm{~b}$ & $77.2 \mathrm{~b}$ & $79.3 \mathrm{~b}$ \\
\hline TSKC $\times$ CTTR-029 & $0 \mathrm{c}$ & $3.3 \mathrm{~d}$ & $14.7 \mathrm{e}$ & $68.5 \mathrm{~b}$ & $79.9 \mathrm{~b}$ & $81.5 \mathrm{~b}$ \\
\hline TSKC $\times$ TRBK -006 & $0 \mathrm{c}$ & $1.7 \mathrm{~d}$ & $30.1 \mathrm{~d}$ & $69.6 \mathrm{~b}$ & $76.6 \mathrm{~b}$ & $76.7 \mathrm{c}$ \\
\hline TSK $\times$ TRBK-CO & $0.6 \mathrm{~b}$ & $3.3 \mathrm{~d}$ & $8.9 \mathrm{f}$ & $54.4 \mathrm{c}$ & $60.0 \mathrm{c}$ & $62.2 \mathrm{c}$ \\
\hline $\mathrm{TSKC} \times(\mathrm{LCR} \times \mathrm{TR})-001$ & $0 \mathrm{c}$ & $0 \mathrm{e}$ & $10.0 \mathrm{e}$ & $42.8 \mathrm{~d}$ & $45.0 \mathrm{~d}$ & $48.3 \mathrm{~d}$ \\
\hline $\mathrm{TSKC} \times(\mathrm{LCR} \times \mathrm{TR})-020$ & $0 \mathrm{c}$ & $6.7 \mathrm{c}$ & $16.1 \mathrm{e}$ & $56.1 \mathrm{c}$ & $66.7 \mathrm{c}$ & $73.9 \mathrm{c}$ \\
\hline $\mathrm{TSKC} \times(\mathrm{LCR} \times \mathrm{TR})-040$ & $0 \mathrm{c}$ & $2.2 \mathrm{~d}$ & $13.9 \mathrm{e}$ & $46.7 \mathrm{c}$ & $52.8 \mathrm{~d}$ & $53.3 \mathrm{~d}$ \\
\hline $\mathrm{TSKC} \times(\mathrm{LCR} \times \mathrm{TR})-059$ & $0.5 \mathrm{~b}$ & $6.5 \mathrm{c}$ & $23.4 \mathrm{~d}$ & $64.1 \mathrm{~b}$ & $68.5 \mathrm{c}$ & $69.0 \mathrm{c}$ \\
\hline $\mathrm{TSKC} \times(\mathrm{LCR} \times \mathrm{TR})-073$ & $0 \mathrm{c}$ & $2.5 \mathrm{~d}$ & $27.9 \mathrm{~d}$ & $58.3 \mathrm{c}$ & $62.8 \mathrm{c}$ & $61.8 \mathrm{c}$ \\
\hline TSKFL $\times$ CTSW-004 & $0 \mathrm{c}$ & $0 \mathrm{e}$ & $4.9 \mathrm{f}$ & $29.6 \mathrm{e}$ & $51.1 \mathrm{~d}$ & $56.0 \mathrm{~d}$ \\
\hline $\mathrm{LRF} \times(\mathrm{LCR} \times \mathrm{TR})-005$ & $0.6 \mathrm{~b}$ & $6.7 \mathrm{c}$ & $13.3 \mathrm{e}$ & $55.6 \mathrm{c}$ & $74.4 \mathrm{c}$ & $80.0 \mathrm{~b}$ \\
\hline LCR $\times$ CTSW-009 & $0 \mathrm{c}$ & $1.1 \mathrm{e}$ & $7.2 \mathrm{f}$ & $51.7 \mathrm{c}$ & $66.7 \mathrm{c}$ & $75.0 \mathrm{c}$ \\
\hline $\mathrm{LCR} \times \mathrm{TR}-001$ & $0 \mathrm{c}$ & $0 \mathrm{e}$ & $2.2 \mathrm{~g}$ & $8.3 \mathrm{~g}$ & $20.6 \mathrm{e}$ & $24.4 \mathrm{f}$ \\
\hline $\mathrm{LVK} \times \mathrm{LCR}-038$ & $0 \mathrm{c}$ & $3.9 \mathrm{~d}$ & $22.2 \mathrm{~d}$ & $62.8 \mathrm{~b}$ & $67.2 \mathrm{c}$ & $68.3 \mathrm{c}$ \\
\hline LVK $\times$ LVA-009 & $0 \mathrm{c}$ & $0 \mathrm{e}$ & $1.6 \mathrm{~g}$ & $29.9 \mathrm{e}$ & $51.6 \mathrm{~d}$ & $59.2 \mathrm{~d}$ \\
\hline HTR-051 & $0 \mathrm{c}$ & $0 \mathrm{e}$ & $6.1 \mathrm{f}$ & $50.8 \mathrm{c}$ & $65.9 \mathrm{c}$ & $75.0 \mathrm{c}$ \\
\hline HTR-053 & $0 \mathrm{c}$ & $9.4 \mathrm{c}$ & $16.7 \mathrm{e}$ & $30.3 \mathrm{e}$ & $32.3 \mathrm{e}$ & $34.4 \mathrm{e}$ \\
\hline HTR-069 & $0 \mathrm{c}$ & $0 \mathrm{e}$ & $2.7 \mathrm{~g}$ & $33.7 \mathrm{e}$ & $58.2 \mathrm{~d}$ & $66.9 \mathrm{c}$ \\
\hline HTR-116 & $0 \mathrm{c}$ & $2.7 \mathrm{~d}$ & $5.1 \mathrm{f}$ & $25.0 \mathrm{e}$ & $48.7 \mathrm{~d}$ & $71.0 \mathrm{c}$ \\
\hline HTR-207 & $0 \mathrm{c}$ & $3.3 \mathrm{~d}$ & $7.1 \mathrm{f}$ & $53.8 \mathrm{c}$ & $69.0 \mathrm{c}$ & $83.2 \mathrm{~b}$ \\
\hline HTR-208 & $0 \mathrm{c}$ & $13.3 \mathrm{c}$ & $26.7 \mathrm{~d}$ & $67.2 \mathrm{~b}$ & $73.3 \mathrm{c}$ & $82.8 \mathrm{~b}$ \\
\hline MXWL $\times$ LHA-001 & $0 \mathrm{c}$ & $2.7 \mathrm{~d}$ & $6.5 \mathrm{f}$ & $34.8 \mathrm{e}$ & $46.2 \mathrm{~d}$ & $52.7 \mathrm{~d}$ \\
\hline $\mathrm{CLEO} \times \mathrm{TRNB}-245$ & $0 \mathrm{c}$ & $2.5 \mathrm{~d}$ & $7.6 \mathrm{f}$ & $28.4 \mathrm{e}$ & $63.2 \mathrm{c}$ & $68.3 \mathrm{c}$ \\
\hline $\mathrm{CLEO} \times \mathrm{TRSW}-287$ & $0.6 \mathrm{~b}$ & $4.4 \mathrm{c}$ & $10.0 \mathrm{e}$ & $31.1 \mathrm{e}$ & $52.8 \mathrm{~d}$ & $58.9 \mathrm{~d}$ \\
\hline Citrandarin 'Indio' & $2.2 \mathrm{~b}$ & $8.2 \mathrm{c}$ & $17.9 \mathrm{e}$ & $68.5 \mathrm{~b}$ & $82.1 \mathrm{~b}$ & $85.9 \mathrm{~b}$ \\
\hline Citrandarin 'Riverside' & $0 \mathrm{c}$ & $5.5 \mathrm{c}$ & $9.8 \mathrm{e}$ & $45.1 \mathrm{~d}$ & $68.4 \mathrm{c}$ & $72.8 \mathrm{c}$ \\
\hline Citrandarin 'San Diego' & $0 \mathrm{c}$ & $3.3 \mathrm{~d}$ & $6.7 \mathrm{f}$ & $31.7 \mathrm{e}$ & $41.7 \mathrm{~d}$ & $48.3 \mathrm{~d}$ \\
\hline Citrange 'Fepagro C-13' & $1.1 \mathrm{~b}$ & $4.9 \mathrm{c}$ & $17.4 \mathrm{e}$ & $52.2 \mathrm{c}$ & $67.4 \mathrm{c}$ & $75.0 \mathrm{c}$ \\
\hline Citrumelo 'Swingle' & $0.5 \mathrm{~b}$ & $21.4 \mathrm{~b}$ & $38.0 \mathrm{c}$ & $58.9 \mathrm{c}$ & $66.0 \mathrm{c}$ & $66.1 \mathrm{c}$ \\
\hline Trifoliata & $14.3 \mathrm{a}$ & $72.8 \mathrm{a}$ & $92.4 \mathrm{a}$ & $92.4 \mathrm{a}$ & $92.4 \mathrm{a}$ & $92.4 \mathrm{a}$ \\
\hline Tangerine tree 'Sunki Tropical' & $0 \mathrm{c}$ & $1.1 \mathrm{~d}$ & $23.3 \mathrm{~d}$ & $61.1 \mathrm{~b}$ & $64.4 \mathrm{c}$ & $66.1 \mathrm{c}$ \\
\hline Lemon tree 'Cravo' & $0.9 \mathrm{~b}$ & $27.2 \mathrm{~b}$ & $57.6 \mathrm{~b}$ & $80.4 \mathrm{a}$ & $83.0 \mathrm{~b}$ & $83.9 \mathrm{~b}$ \\
\hline CV $(\%)$ & 182.6 & 44.8 & 19.4 & 11.2 & 10.4 & 8.9 \\
\hline $\mathrm{F}$ & $7.03 * *$ & $18.25^{* *}$ & $29.58 * *$ & $24.01 * *$ & $16.49^{* *}$ & $19.37^{* *}$ \\
\hline
\end{tabular}

Note. $* \mathrm{e}^{* *}$ meaningful at $5 \%$ and $1 \%$ probability, respectively, by the $\mathrm{F}$ test. Means followed by the same letter in the column belong to the same group by the Scott-Knott's clustering test $(\mathrm{p} \leq 0.05)$.

${ }^{1}$ Abbreviations: TSKC-tangerine tree 'Sunki' [Citrus sunki (Hayata) hort. ex Tanaka common; CTSW-citrumelo 'Swingle' (C. paradisi Macfad. $\times$ Poncirus trifoliata (L.) Raf.]; TRFD-P. trifoliata 'Flying Dragon'; CTCM-citrange 'Coleman'; CTTR-citrange $[C$. sinensis $($ L.) Osbeck $\times$ P. trifoliata $]$ 'Troyer'; TRBK-P. trifoliata 'Benecke'; LCR-lemon tree 'Cravo' (C. limonia Osbeck); TR-P. trifoliata; TSKFL-tangerine tree 'Sunki da Flórida'; LRF-lemon tree 'Rugoso da Flórida' (C. jambhiri Lush.); LVK-lemon tree 'Volkameriano' (C. volkameriana V. Ten. \& Pasq.; LVA-orange tree 'Valência' (C. sinensis); HTR-trifoliata hybrid; MXWL-mandarin orange tree 'Willow Leaf' (C. deliciosa Tenore); LHA-orange tree 'Hamlin' $(C$. sinensis); CLEO-tangerine tree 'Cleópatra' (C. reshni hort. ex Tanaka); TRNB-P. trifoliata 'Barnes'; TRSW-P. trifoliata 'Swingle'. 
The highest emergence speed index (ESI) in the first period was observed in Trifoliata (Table 3). This rootstock completed seedling emergence 50 days after sowing (DAS). Besides Trifoliata, other rootstocks showed uniformity in seedling emergence in the first period (0-56 DAS), such as lemon tree 'Cravo' and citrumelo 'Swingle' (Table 3). Among rootstocks that are precocious regarding emergence, lemon tree 'Cravo' usually has this behavior, as observed by Sousa et al. (2002) and Rodrigues et al. (2015).

Table 3. Linear equations and emergence speed index (ESI) at the seed emergence period, divided in initial period (0-56 DAS) and final period (57-98 DAS)

\begin{tabular}{|c|c|c|c|c|c|c|}
\hline \multirow{2}{*}{ Rootstock } & \multicolumn{2}{|l|}{ All period } & \multicolumn{2}{|c|}{ Jun 1 to Jul 8 (56 DAS) } & \multicolumn{2}{|c|}{ Jul 11 to Aug 19 (98 DAS) } \\
\hline & Linear equation & ESI & Linear equation & ESI & Linear equation & ESI \\
\hline $\mathrm{TSKC} \times \mathrm{CTSW}-025$ & $y=1.4811 x-6352$ & 20.14 & $y=1.6652 x-7143$ & 4.43 & $y=0.164 x-6957$ & 15.61 \\
\hline TSKC $\times$ CTSW -028 & $y=0.7154 x-3068$ & 8.04 & $y=0.5165 x-2215$ & 1.20 & $y=0.3408 x-1459$ & 11.45 \\
\hline TSKC $\times$ CTSW -031 & $y=0.7784 x-3337$ & 13.33 & $y=1.0906 x-4677$ & 4.28 & $y=0.1091 x-4634$ & 3.52 \\
\hline TSKC $\times$ CTSW -033 & $y=0.9959 x-4272$ & 10.81 & $y=0.3627 x-1555$ & 1.78 & $y=0.8563 x-3672$ & 18.33 \\
\hline TSKC $\times$ CTSW -036 & $y=0.5265 x-2258$ & 4.80 & $y=0.1775 x-7615$ & 0.35 & $y=0.5124 x-2198$ & 11.65 \\
\hline TSKC $\times$ CTSW -041 & $y=1.4984 x-6426$ & 21.24 & $y=1.4798 x-6347$ & 5.17 & $y=0.3317 x-1415$ & 17.99 \\
\hline TSKC $\times$ TRFD-003 & $\mathrm{y}=1.345 \mathrm{x}-5768$ & 20.37 & $y=1.7532 x-7520$ & 5.53 & $y=0.186 x-7906$ & 10.04 \\
\hline TSKC $\times$ TRFD -006 & $y=1.2767 x-5476$ & 17.04 & $y=0.6972 x-2990$ & 3.83 & $y=0.3416 x-1459$ & 12.53 \\
\hline TSKC $\times$ TRFD-007 & $y=1.1445 x-4909$ & 14.01 & $y=0.9298 x-3988$ & 3.01 & $y=0.8166 x-3500$ & 14.78 \\
\hline TSKC $\times$ CTCM-008 & $y=0.2986 x-1280$ & 2.63 & $y=0.0956 x-4101$ & 0.24 & $y=0.3875 x-1662$ & 6.14 \\
\hline TSKC $\times$ CTTR-002 & $y=1.2613 x-5409$ & 17.84 & $\mathrm{y}=1.1959 \mathrm{x}-5129$ & 4.59 & $y=0.5236 x-2241$ & 12.29 \\
\hline TSKC $\times$ CTTR -029 & $y=1.3712 x-5881$ & 16.06 & $y=0.9135 x-3918$ & 2.63 & $y=0.6044 x-2588$ & 25.06 \\
\hline TSKC $\times$ TRBK -006 & $y=1.2767 x-5476$ & 17.04 & $y=0.6972 x-2990$ & 3.83 & $\mathrm{y}=0.3416 \mathrm{x}-1459$ & 12.53 \\
\hline TSK $\times$ TRBK-CO & $y=1.0373 x-4449$ & 12.88 & $y=0.7409 x-3178$ & 2.37 & $y=0.3362 x-1438$ & 14.79 \\
\hline $\mathrm{TSKC} \times(\mathrm{LCR} \times \mathrm{TR})-001$ & $y=0.8126 x-3485$ & 9.41 & $y=0.6438 x-2761$ & 1.42 & $y=0.2527 x-1081$ & 10.11 \\
\hline $\mathrm{TSKC} \times(\mathrm{LCR} \times \mathrm{TR})-020$ & $y=1.1409 x-4893$ & 14.43 & $y=0.7865 x-3373$ & 3.10 & $y=0.672 x-2879$ & 19.42 \\
\hline $\mathrm{TSKC} \times(\mathrm{LCR} \times \mathrm{TR})-040$ & $y=0.901 x-3864$ & 10.91 & $y=0.7142 x-3063$ & 1.96 & $y=0.3476 x-1487$ & 14.31 \\
\hline $\mathrm{TSKC} \times(\mathrm{LCR} \times \mathrm{TR})-059$ & $y=1.1088 x-4755$ & 15.92 & $y=1.0923 x-4685$ & 4.02 & $y=0.2962 x-1265$ & 14.62 \\
\hline $\mathrm{TSKC} \times(\mathrm{LCR} \times \mathrm{TR})-073$ & $y=1.0472 x-4491$ & 14.57 & $y=0.5673 x-2433$ & 3.44 & $y=0.1623 x-6909$ & 7.90 \\
\hline TSKFL $\times$ CTSW-004 & $y=0.8896 x-3816$ & 8.00 & $y=1.2571 x-5392$ & 0.64 & $y=0.9826 x-4215$ & 19.00 \\
\hline $\mathrm{LRF} \times(\mathrm{LCR} \times \mathrm{TR})-005$ & $y=1.2761 x-5474$ & 14.58 & $y=0.6459 x-2770$ & 2.63 & $y=1.0159 x-4356$ & 26.28 \\
\hline LCR $\times$ CTSW-009 & $\mathrm{y}=1.2331 \mathrm{x}-5290$ & 12.36 & $y=0.5142 x-2205$ & 1.27 & $y=0.9109 x-3905$ & 26.22 \\
\hline $\mathrm{LCR} \times \mathrm{TR}-001$ & $y=0.3596 x-1542$ & 2.98 & $y=0.0912 x-3911$ & 0.27 & $y=0.5702 x-2447$ & 7.76 \\
\hline LVK $\times$ LCR -038 & $y=1.1407 x-4892$ & 14.79 & $y=1.0386 x-4454$ & 3.04 & $y=0.3199 x-1367$ & 18.09 \\
\hline LVK $\times$ LVA-009 & $y=0.9576 x-4108$ & 7.96 & $y=0.1132 x-4855$ & 0.25 & $y=1.1228 x-4817$ & 26.53 \\
\hline HTR-051 & $y=1.2189 x-5228$ & 11.97 & $y=0.4866 x-2087$ & 1.07 & $y=0.883 x-3786$ & 24.07 \\
\hline HTR-053 & $y=0.49 x-2101$ & 8.93 & $y=0.7134 x-3059$ & 3.14 & $y=0.148 x-6326$ & 2.73 \\
\hline HTR-069 & $y=1.0674 x-4579$ & 8.84 & $y=0.1649 x-7071$ & 0.39 & $y=1.3605 x-5837$ & 27.26 \\
\hline HTR-116 & $y=1.0071 x-4320$ & 8.91 & $y=0.2565 x-1100$ & 1.02 & $y=1.4929 x-6407$ & 20.01 \\
\hline HTR-207 & $y=1.2946 x-5553$ & 13.00 & $y=0.4587 x-1967$ & 1.64 & $y=1.2454 x-5342$ & 26.35 \\
\hline HTR-208 & $y=1.2074 x-5178$ & 18.53 & $y=1.1769 x-5047$ & 5.45 & $y=0.5897 x-2525$ & 18.19 \\
\hline MXWL $\times$ LHA-001 & $y=0.8158 x-3499$ & 8.59 & $y=0.3163 x-1356$ & 1.23 & $y=0.7119 x-3053$ & 19.31 \\
\hline CLEO $\times$ TRNB -245 & $\mathrm{y}=1.0626 \mathrm{x}-4558$ & 9.87 & $y=0.3427 x-1469$ & 1.27 & $y=1.4354 x-6159$ & 21.83 \\
\hline CLEO $\times$ TRSW -287 & $y=0.8932 x-3831$ & 9.84 & $y=0.3627 x-1555$ & 2.00 & $y=1.0663 x-4575$ & 18.94 \\
\hline Citrandarin 'Indio' & $y=1.3481 x-5782$ & 17.56 & $y=0.8481 x-3637$ & 3.97 & $y=0.7796 x-3340$ & 26.52 \\
\hline Citrandarin 'Riverside' & $y=1.177 x-5048$ & 13.14 & $y=0.4546 x-1949$ & 2.16 & $y=0.9277 x-3977$ & 29.16 \\
\hline Citrandarin 'San Diego' & $y=0.7538 x-3233$ & 8.45 & $\mathrm{y}=0.3479 \mathrm{x}-1492$ & 1.42 & $y=0.5958 x-2555$ & 16.93 \\
\hline Citrange 'Fepagro C-13' & $y=1.1508 x-4936$ & 14.48 & $y=0.8135 x-3489$ & 3.22 & $y=0.7886 x-3380$ & 18.19 \\
\hline Citrumelo 'Swingle' & $\mathrm{y}=0.8951 \mathrm{x}-3838$ & 18.94 & $y=1.2509 x-5364$ & 7.51 & $\mathrm{y}=0.3019 \mathrm{x}-1290$ & 11.14 \\
\hline Trifoliata & $y=0.7387 x-3162$ & 38.85 & $y=2.3194 x-9945$ & 21.96 & $\mathrm{y}=0.000 x-92.41$ & 0.00 \\
\hline Tangerine tree 'Sunki Tropical' & $y=1.1088 x-4755$ & 14.57 & $y=1.2424 x-5329$ & 3.09 & $y=0.2133 x-9095$ & 5.60 \\
\hline Lemon tree 'Cravo' & $y=1.1579 x-4964$ & 24.62 & $y=2.1012 x-9012$ & 9.71 & $y=0.1707 x-7250$ & 9.84 \\
\hline
\end{tabular}

Note. ${ }^{1}$ Abbreviations: TSKC-tangerine tree 'Sunki' [Citrus sunki (Hayata) hort. ex Tanaka common; CTSW-citrumelo 'Swingle' (C. paradisi Macfad. $\times$ Poncirus trifoliata (L.) Raf.]; TRFD-P. trifoliata 'Flying Dragon'; CTCM-citrange 'Coleman'; CTTR-citrange [C. sinensis (L.) Osbeck $\times P$. trifoliata] 'Troyer'; TRBK- $P$. trifoliata 'Benecke'; LCR-lemon tree 'Cravo' (C. limonia Osbeck); TR-P. trifoliata; TSKFL-tangerine tree 'Sunki da Flórida'; LRF-lemon tree 'Rugoso da Flórida' (C. jambhiri Lush.); LVK-lemon tree 'Volkameriano' 
(C. volkameriana V. Ten. \& Pasq.; LVA-orange tree 'Valência' (C. sinensis); HTR-trifoliata hybrid; MXWL-mandarin orange tree 'Willow Leaf' $(C$. deliciosa Tenore); LHA-orange tree 'Hamlin' ( $C$. sinensis); CLEO-tangerine tree 'Cleópatra' (C. reshni hort. ex Tanaka); TRNB-P. trifoliata 'Barnes'; TRSW-P. trifoliata 'Swingle'.

The comparison among rootstocks which are commercially used in Brazilian citriculture (lemon tree 'Cravo', Trifoliata, citrumelo 'Swingle' and tangerine tree 'Sunki Tropical') and hybrids whose parents are the tangerine tree 'Sunki', Trifoliata and lemon tree 'Cravo', showed that Trifoliata and lemon tree 'Cravo' exhibited the highest emergence percentages (Figure 3). Rodrigues et al. (2015) reported that 'Sunki Tropical' had higher values than other rootstocks they evaluated, since this tangerine tree exhibited $100 \%$ of seedling emergence. Results reported by this paper are very different, i.e., this rootstock had $66.1 \%$ of seedling emergence (Table 2 ). Since both studies used the same substrate and the same methodology of sowing and irrigation, the genotype 'Sunki Tropical' may not have exhibited good seedling emergence either due to local characteristics or because of the quality of the seeds, considering that other studies showed good seedling emergence.

When hybrids were grouped with their parents-tangerine tree 'Sunki', lemon tree 'Cravo' and Trifoliata-the highest seedling emergence was found in the group of hybrids which has Trifoliata in its genetic constitution, either directly or indirectly. This group was 5\% above the others (Figure 3). It means that the genotype Trifoliata may pass, to its descendants, characteristics that enable high adaptation to the local weather, since it had the highest emergence of all genotypes.

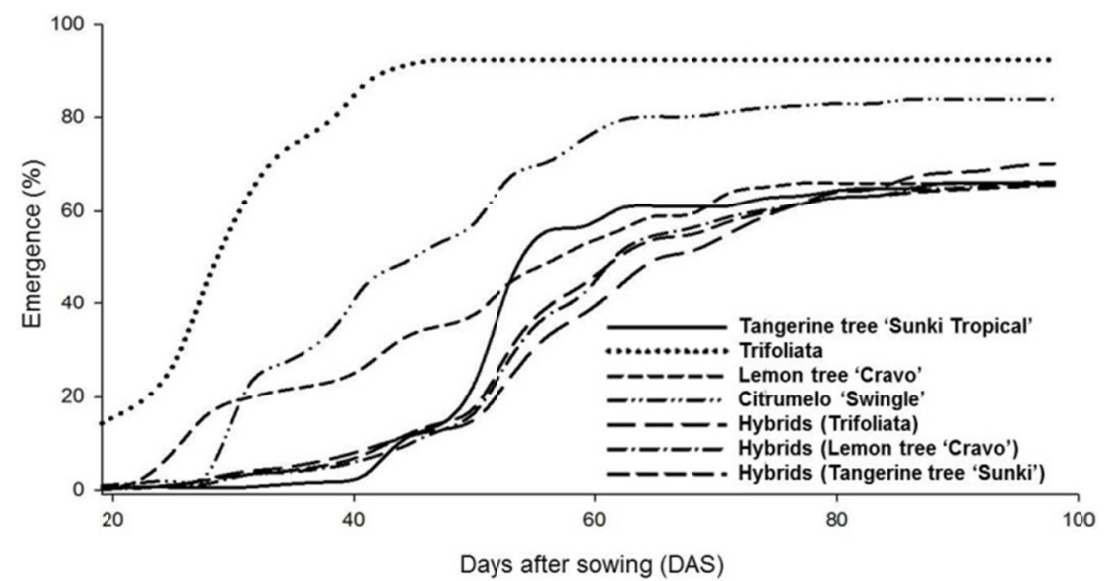

Figure 3. Emergence time of rootstocks Trifoliata (P. trifoliata), tangerine tree 'Sunki Tropical'[Citrus sunki (Hayata) hort. ex Tanaka], lemon tree 'Cravo' (C. limonia Osbeck), citrumelo 'Swingle' (C. paradisi Macfad. $\times$ P. trifoliata) and groups of parental hybrids: Trifoliata, lemon tree 'Cravo' and tangerine tree 'Sunki'

\section{Conclusion}

Final values of seedling emergence of Trifoliata and both hybrids TSKC $\times$ CTSW-041 and TSKC $\times$ CTSW-025 were higher than the ones of the other rootstocks under study. Trifoliata needed the lowest period of time to complete seedling emergency but the lemon tree 'Cravo' also stands out, regarding this variable. Genotpes under study needed 98 days after sowing to complete seedling emergence.

\section{References}

FAO-FAOSTAT. (2014). Food and Agriculture Organization of the United Nations. Retrieved from http://faostat3.fao.org/download/Q/QC/E

Girardi, E. A., Stuchi, E. S., Passos, O. S., Soares Filho, W. S., Parolin, L. G., Reiff, E. T., ... Dobre, R. P. (2017). Minimudas: Produção de mudas de citros em recipientes pequenos (Embrapa Mandioca e Fruticultura, Circular Técnica, 123). Cruz das Almas, BA: Embrapa Mandioca e Fruticultura.

Hayashi, S., Girardi, E. A., Silva, S. R., Stuchi, E. S., \& Cantuarias-Avilés, T. E. (2012). Avaliação de fita fotodegradável para enxertia de mudas de citros. Revista Brasileira de Fruticultura, 32(2), $641-645$. https://doi.org/10.1590/S0100-29452012000200042 
Maguire, J. D. (1962). Speed of germination-aid in selection and evaluation for seedling emergence and vigor. Crop Science, 2(2), 176-177. https://doi.org/10.2135/cropsci1962.0011183X000200020033x

Oliveira, R. P., \& Scivittaro, W. B. (2007). Formação do porta-enxerto Trifoliata: Época de semeadura. Ciência Rural, 37(1), 281-283. https://doi.org/10.1590/S0103-84782007000100047

Oliveira, R. P., Soares Filho, W. S., Passos, O. S., Scivittaro, W. B., \& Rocha, P. S. G. (2008). Porta-enxertos para citros (Embrapa Clima Temperado, Documentos, 226). Pelotas, RS: Embrapa Clima Temperado.

Oliveira, R. P., Ueno, B., Scivittaro, W. B., Carvalho, F. L. C., Petry, H. B., \& Moreno, M. B. (2016). Produção de Sementes de Porta-enxertos de Citros (Embrapa Clima Temperado, Documentos, 417). Pelotas, RS: Embrapa Clima Temperado.

Oliveira, R. P., Scivittaro, W. B., Carvalho, F. L. C., Souza, P. V. D., Tarillo, V. R. C., \& Lima, G. A. S. (2017). Produção de Mudas de Citros em Ambiente Protegido (Embrapa Clima Temperado, Documentos, 444). Pelotas, RS: Embrapa Clima Temperado.

Parolin, L. G., Girardi, E. A., Stuchi, E. S., Costa, D. P., Jesus, C. A. S., Reiff, E. T., ... Soares Filho, W. S. (2017). Produção de mudas de citros em viveiro protegido, utilizando diferentes combinações de copa e de porta-enxerto (Embrapa Mandioca e Fruticultura, Documentos, 84), Cruz das Almas, BA: Embrapa Mandioca e Fruticultura.

Passos, O. S., Peixouto, L. S., \& Santos, L. C. (2006). Caracterização de híbridos de Poncirus trifoliata e de outros porta-enxertos de citros no estado da Bahia. Revista Brasileira de Fruticultura, 28(3), 410-413. https://doi.org/10.1590/S0100-29452006000300016

Petry, H. B., Reis, B., Silva, R. R., Gonzatto, M. P., \& Schwarz, S. F. (2015). Porta-enxertos influenciam o desempenho produtivo de laranjeiras-de-umbigo submetidas a poda drástica. Pesquisa Agropecuária Tropical, 45(4), 449-455. https://doi.org/10.1590/1983-40632015v4537005

Rodrigues, M. J. S., Ledo, C. A. S., Girardi, E. A., Almeida, L. A. H., \& Soares Filho, W. S. (2015). Caracterização de frutos e propagação de porta-enxertos híbridos de citros em ambiente protegido. Revista Brasileira de Fruticultura, 37(2), 457-470. https://doi.org/10.1590/0100-2945-068/14

Sarmiento, A. I. P., Giuliani, J. C., \& Souza, P. V. D. (2016). Morfologia de frutos e sementes de porta-enxertos de citros cultivados em ambiente protegido. Revista U.D.C.A. Actualidad \& Divulgación Científica, 19(1), 17-24. https://doi.org/10.31910/rudca.v19.n1.2016.106

Sousa, H. U., Ramos, J. D., Pasqual, M., \& Ferreira, E. A. (2002). Efeito do ácido giberélico sobre a germinação de sementes de porta-enxertos cítricos. Revista Brasileira de Fruticultura, 24(2), 496-499. https://doi.org/ $10.1590 / \mathrm{S} 0100-29452002000200043$

Teixeira, P. T. L., Schäfler, G.., Souza, P. V. D., \& Todeschini, A. A. (2009). Escarificação química e o desenvolvimento inicial de porta-enxertos cítricos. Revista Brasileira de Fruticultura, 31(3), 865-871. https://doi.org/10.1590/S0100-29452009000300033

Wrege, M. S., Oliveira, R. P., João, P. L., Herter, F. G., Steinmetz, S., Reisser Júnior, C., ... Pereira, I. S. (2004). Zoneamento agroclimático para a cultura dos citros no Rio Grande do Sul (Embrapa Clima Temperado, Documentos 117). Pelotas, RS: Embrapa Clima Temperado.

\section{Copyrights}

Copyright for this article is retained by the author(s), with first publication rights granted to the journal.

This is an open-access article distributed under the terms and conditions of the Creative Commons Attribution license (http://creativecommons.org/licenses/by/4.0/). 